\title{
The Prediction and Theoretical Study for Chemical Reactivity, Thermophysical and Biological Activity of Morpholinium Nitrate and Nitrite Ionic Liquid Crystals: A DFT Study
}

\author{
Mohammad Jahidul Islam a (D), Ajoy Kumer b,* (D), Nuruzzaman Sarker a (D), Sunanda Paul c (D), \\ Afroza Zannat ${ }^{\mathrm{b}}$ \\ a Department of Physics, European University of Bangladesh, Dhaka-1216, Bangladesh \\ ${ }^{b}$ Department of Chemistry, European University of Bangladesh, Dhaka-1216, Bangladesh \\ ${ }^{c}$ Department of Biochemistry and Molecular Biology, University of Chittagong, Chittagong,Hathazari-4334, \\ Bangladesh
}

\section{A R T I C L E I N F O \\ Received: 03 April 2019 \\ Revised: 22 April 2019 \\ Accepted: 02 May 2019 \\ Available online: 04 May 2019 \\ DOI: $10.33945 /$ SAMI/AJCA.2019.4.5}

\section{K E Y W O R D S}

Morpholine

Ionic Liquids

QSAR

HOMO

LUMO

Vibrational spectroscopy

\section{A B S T R A C T}

As the morpholine and morphine have been used all over the world as pain killer drugs even used in cancer treatment, so the morpholine is more demanding chemical molecule. In our work, the morpholine has included the addition of inorganic anions like nitrate and nitrite for forming morpholinium based Ionic Liquid. Their chemical properties, biochemical properties, and physio-chemical properties are evaluated using computational chemistry through the Density Functional Theory (DFT). The biological properties have been shown that biological activity in the designed ionic liquid for uses in new drug discovery. From QSAR study, the value of the Log P is 0.713 and 1.7 which indicates hydrophobic nature and PIC50 is -2.14 and -3.96 respectively. The nitrate and nitrite comparison have been highlighted through this work. From QSAR and PIC50, it is seen that due to the nitrate addition with morpholine is more biological activity than nitrite. On the other hand, the toxicity of nitrate is less than nitrite.

G RA P H I C A L A B S T RACT

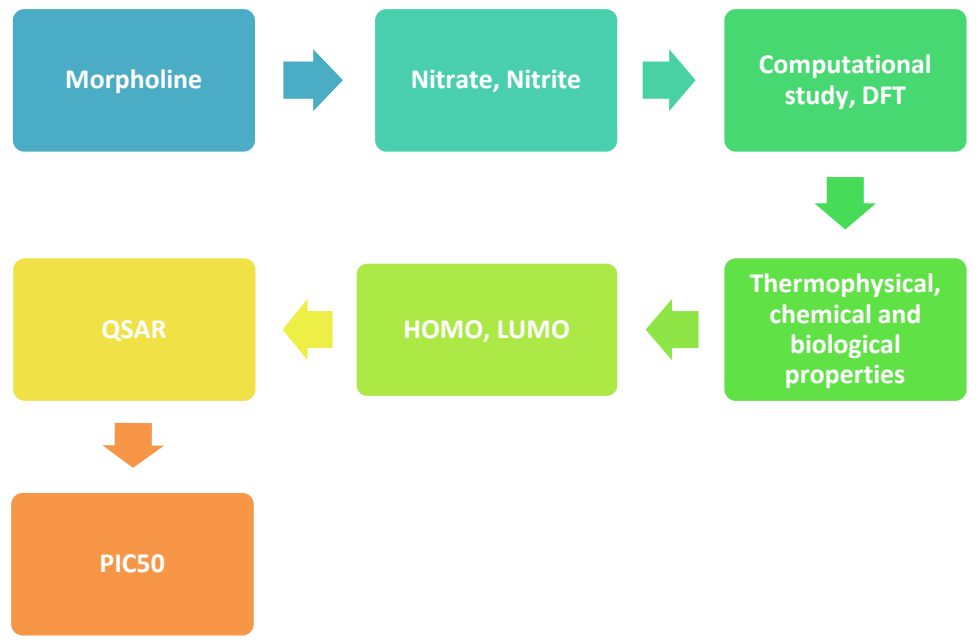

\footnotetext{
* Corresponding author's E-mail address:

kumarajoy.cu@gmail.com
} 


\section{Introduction}

Morphine is recognized as a highly-potent opiate analgesic drug [1] and is used the vital active agent in opium and the prototypical opioid [2]. The animated activity of morphine acts directly on the central nervous system (CNS) to relieve pain, and tolerance, physical and psychological dependences develop very rapidly[3,4]. In the present time, the morphine is used as the prototype narcotic drug so that it is distributed in the human brain and stay long time with high densities in the posterior amygdale, hypothalamus, thalamus, nucleus caudatus, putamen, and certain cortical areas even was found in the spinal cord and in the spinal nucleus of the trigeminal nerve $[5,6]$.

Due to the vital role of morphine in pharmaceutical drug and huge impact on human body, the new term was introduced as morpholinium based Ionic Liquids as designer molecule in engineering even drug discovery [7] due to tunable thermo-physical and chemical properties $[8,9]$. Ionic liquids are the melted salts consisting of discrete organic cation with organic and inorganic anion [10]. Most of the carboxylate anion with ammonium, phosphonium and imazolium ionic liquids shows the high level of biological activity [11]. That is the reason to design new morpholinium Ionic liquids for uses as drug [12-14].

The Density functional theory (DFT) finds increasingly broad application in the chemical and materials sciences for the interpretation and prediction of complex system behavior at an atomic scale [15]. Specifically, DFT computational methods are applied for the study of systems to synthesis and processing parameters .The DFT is used to determine chemical structures of molecules and structural changes in molecules [16]. Especially, B3LYP method is widely used because it yields results very close to experimental results. Moreover, the DFT is a very useful method for predicting the experimental results.

In this study, taking into account all the benefits of designing new drug molecule, to save time and costing, the computational computer programming is used to determine the thermophysical, chemical and biological activity of some morpholinium nitrate and nitrite ILs salts.

A thermodynamic system is a definite macroscopic region or space in the universe in which one or more thermodynamic processes take place. This system has a specific volume consisting of molecules and atoms with continuous movement and concussion by the interaction with the external surrounding. The internal properties and its interaction with the surrounding determine the system behavior [17].

The geometrical parameters and HOMOLUMO energy level of complex were calculated at B3LYP [18]. The B3LYP is considered as a hybrid functional developed in the late 1980 which turns out that DFT and Hartree-Fock based methods are basically trying to do the same thing of recovering electron correlation in very short time with top level of error [19]. On the other hand, the most important work of HOMO, LUMO is to mention the chemical reactivity and region of chemical reactivity. Calculating the HOMO, and LUMO, it is found out that the part of molecule where the electrophillic and neucleophilic can be attached. Their molecular structural relationship, HOMO, LUMO, quantum chemical properties, and Log $P$ plays the role of the chemical reactivity, biological activity and hydrophobicity and hydrophobicity of chemicals in relation with living cells activity and associated mechanistic interactions [20,21].

\section{Computing methods for simulation}

The molecular modeling program permits to build and analyze different molecular structures and determine the 
molecular, electronic, and biological properties. In order to create the spatial chemical structure of each calculated molecule, the two-dimensional structure of the molecule shall be built step-by-step by drawing. Then hydrogen atoms are automatically added. Thereafter, building option and chemical structure is converted into 3D structure. The first step in getting the main characteristic parameters of molecules is to optimize the molecular structure to obtain a configuration characterized by a minimum free energy. In sitting the DFT was fixed via 6G-31G*, and B3-LYP[22]. After completing optimization, the theoretical properties of the studied compound such as free energy, entropy, dipole moment, binding energy, nuclear energy, electronics energy, heat of formation, the HOMO, LUMO are recorded. The QSAR properties of molecules like charge density, surface area grid, volume, Log P, polarizability, refractivity, molecular mass, were calculated. Using the compute in NMR, the coupling, shielding and shielding tensor were determined.

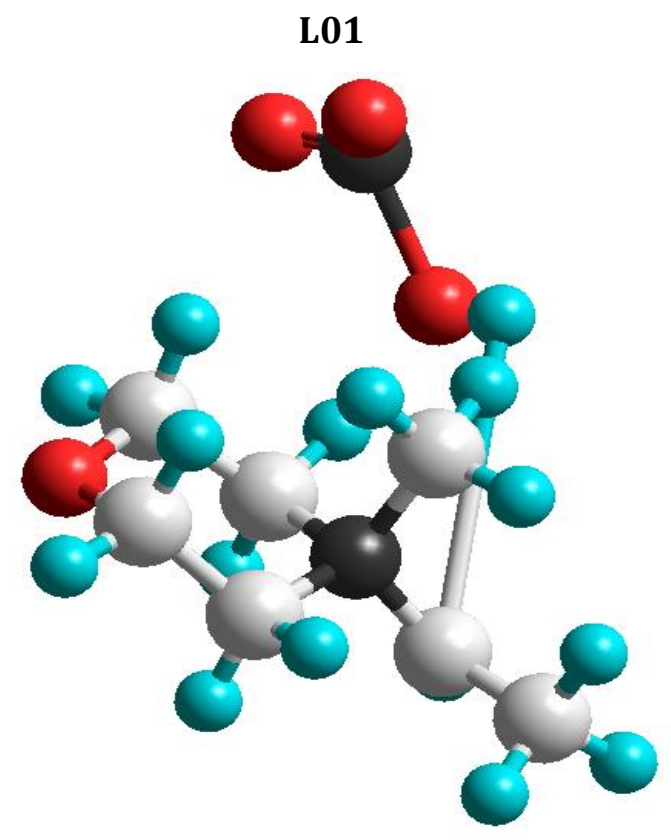

\section{Results and discussions}

\section{Optimized structure}

A representation of the molecular structure optimized which contains the values of the reactivity indices is called the reactive molecular diagram. The optimized structure of optimized molecules using the Hyper Chem 8.0.10 software is represented in Figure 1.

\section{HOMO-LUMO}

The energy levels of the molecular orbitals order HOMO and LUMO ILs give information on the possible electronic transition. The HOMO and LUMO also indicate the electrophilic and nucleophilic attraction region in molecule shown in Figure 2. The LUMO-HOMO gap is the most important parameter for the chemical reactivity. The shorter LUMO-HOMO gap is considered as the high reactivity.

The data of HOMO, LUMO for different energy levels is listed in Table 1.

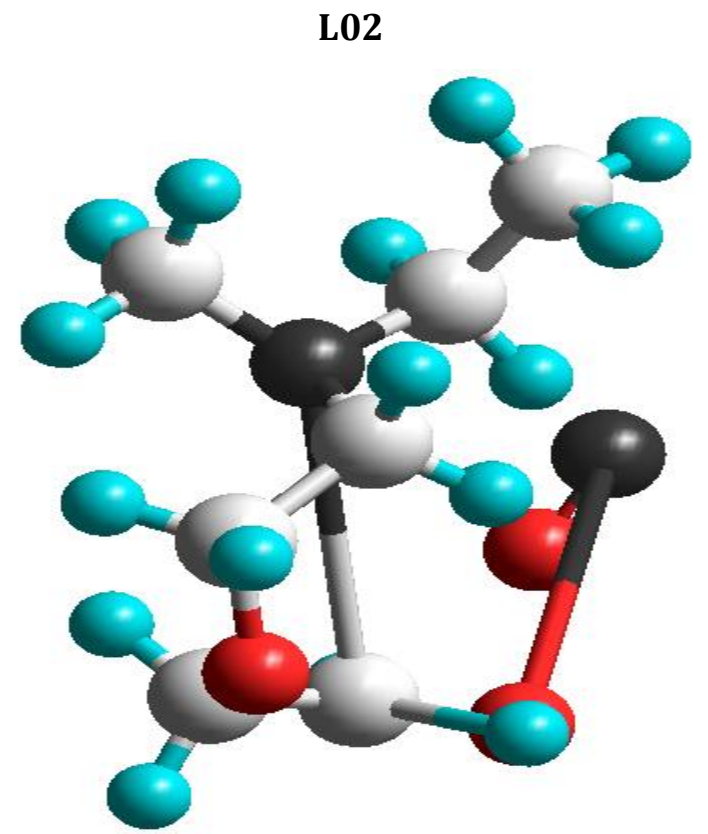

Figure 1. Optimized structure in the cylinder shape, color: red is oxygen, cyan is hydrogen, brown is carbon and black is nitrogen 

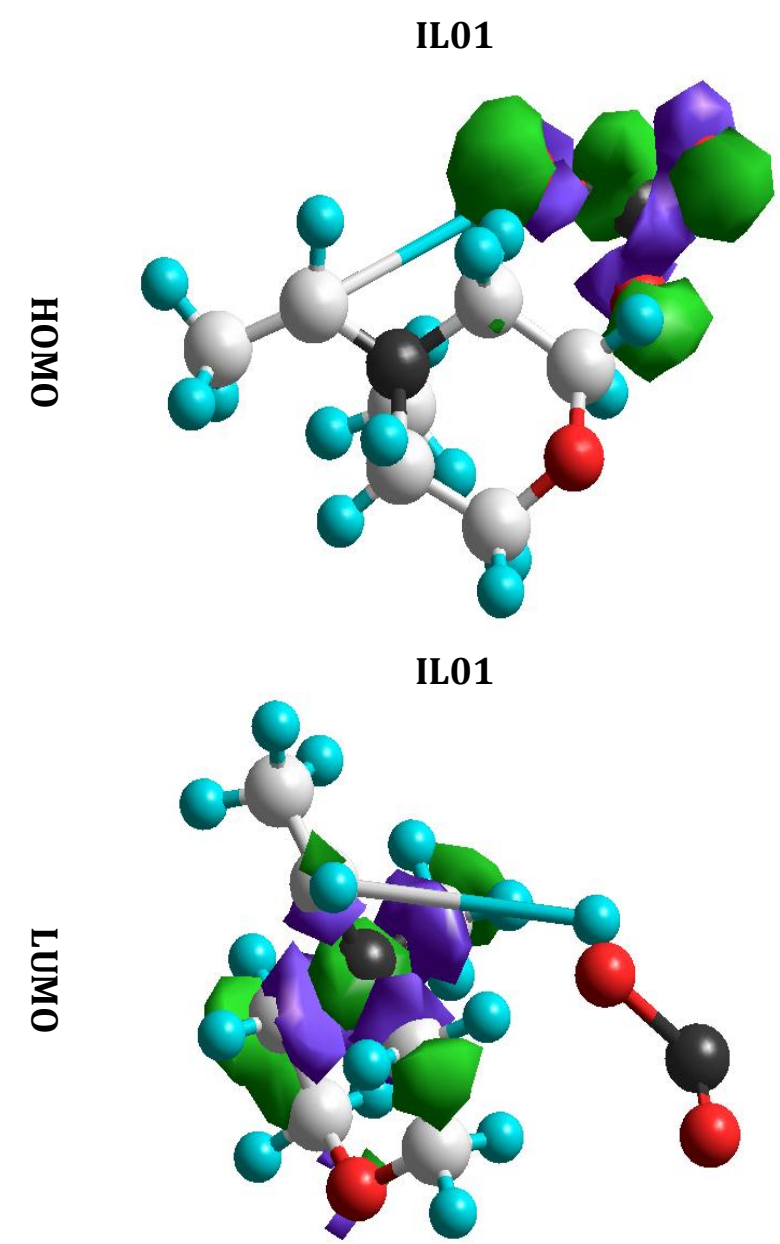

IL02

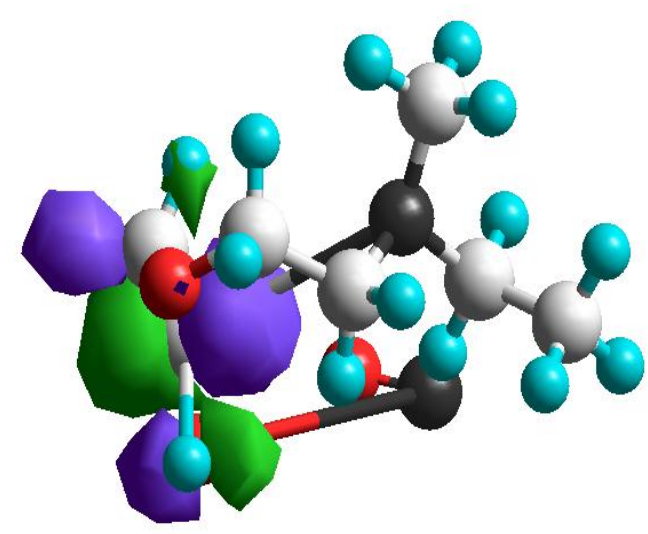

IL02

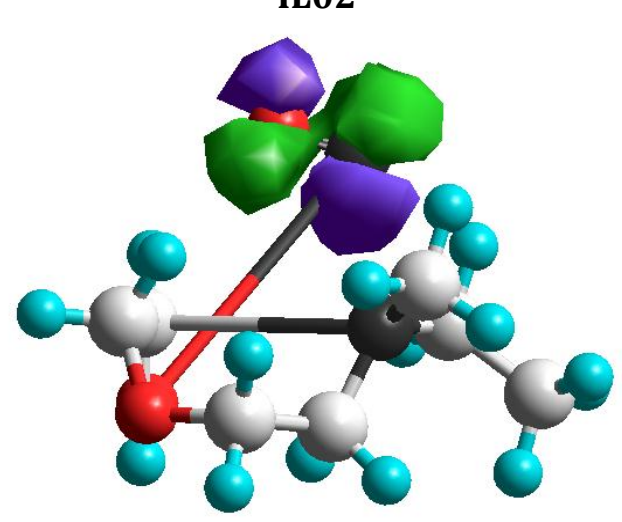

Figure 2. The frontier orbitals: a) HOMO and b) LUMO

Table 1. Data for HOMO LUMO

\begin{tabular}{ccc} 
& IL01 & IL02 \\
HOMO(0), eV & -3.6846 & -8.2785 \\
HOMO(-1), eV & 4.7369 & 0.1616 \\
HOMO(-2), eV & 5.3648 & 0.7423 \\
LUMO, (0), eV & 4.7369 & 0.1616 \\
LUMO, (-1), eV & -3.6846 & -8.2785 \\
LUMO, (-2), eV & -3.8562 & -9.1484 \\
\hline
\end{tabular}

Chemical reactivity by DFT calculations

The Energy of the HOMO is directly related to the ionization potential and LUMO Energy is directly related to the electron affinity. Energy difference between HOMO and LUMO orbital is called as energy gap which is an important parameter that determines the stability of the structures. The energy gap is used in determining molecular electrical transport properties. In addition, according to Koopmans' theorem the energy gap, E gap, defined as the difference between HOMO and LUMO 
energy [13].

$$
\mathrm{E}_{\text {gap }}=\left(\mathrm{E}_{\mathrm{LUMO}}-\mathrm{E}_{\mathrm{HOMO}}\right) \approx \mathrm{IP}-\mathrm{EA}
$$

The ionization potential (I) and electron affinity (A) can be estimated from the HOMO and LUMO energy values as following equation no 1 and 2 . The data of HOMO, LUMO gap, ionization potential, and electron affinity are given in Table 2 .

$$
\begin{aligned}
& I=-E_{\text {НОМо }} \\
& A=-E_{\text {LUMO }}
\end{aligned}
$$

Table 2. Data for HOMO, LUMO, IP, EA, and LUMO- HOMO gap ( $\Delta \mathrm{E})$

\begin{tabular}{ccc} 
& ILO1 & IL02 \\
HOMO, (eV) & -3.6846 & -8.2785 \\
LUMO, (eV) & 4.7369 & 0.1616 \\
$\Delta \mathrm{E},($ LUMO-HOMO gap) (eV) & 8.4215 & 8.4401 \\
Ionization potential (I),eV & 3.6846 & 8.2785 \\
Electron affinity (A),eV & -4.7369 & -0.1616 \\
\hline
\end{tabular}

The HOMO and LUMO energies are used for the determination of global reactivity descriptors. It is important that electrophilicity $(\omega)$, chemical potential $(\mu)$, electronegativity $(\chi)$, hardness $(\eta)$ and softness $(S)$ be put into a molecular orbital's framework [12]. We focus on the HOMO and LUMO energies in order to determine the interesting molecular/atomic properties and chemical quantities. These are calculated as following equations given in Table 3.

$$
\begin{aligned}
& (\mu)=-\frac{I+A}{2} \\
& (\eta)=\frac{I-A}{2} \\
& (S)=\frac{1}{\eta} \\
& (\chi)=\frac{I+A}{2} \\
& (\omega)=\frac{\mu^{2}}{2 \eta}
\end{aligned}
$$

\section{Thermo physical properties}

The binding free energy of the optimized molecules is calculated by performing docking process. The molecule with minimum binding energy will have the maximum binding affinity. The binding free energy of the designed molecules is obtained by eliminating the energy of the main molecule. Having the maximum binding affinity, indicating as the best molecule for drug leads molecules targeting computationally. We can find out the drug binding affinity by using the fitness of the drug, which can bind to the target molecule during the docking process and the second way is using Gibbs free energy calculations. According to this more negative value, we can consider a more effective drug. The thermo-physical properties like total energy, entropy, free energy, heat capacity, dipole moment, binding energy, heat of formation, electronic energy and nuclear energy are provided I Table 4.

On the other hand, the entropy and heat capacity are to vary with varying temperature. These properties can be calculate according to vibrational properties of electron as shown in Table 5. 
Table 3. Biological and Chemical reactivity

\begin{tabular}{ccc} 
& IL01 & IL02 \\
Hardness, $(\eta)$ & 4.2107 & 4.0584 \\
Softness, $(\mathrm{S})$ & 0.2374 & 0.2464 \\
Electrophilicity $(\omega)$, & 0.0328 & 2.0292 \\
Chemical potential, $(\mu)$ & 0.5261 & -4.0584 \\
Electronegativity, $(\chi)$ & -0.5261 & 4.0584 \\
Ionization potential $(\mathrm{I}), \mathrm{eV}$ & 3.6846 & 8.2785 \\
Electron affinity $(\mathrm{A}), \mathrm{eV}$ & -4.7369 & -0.1616 \\
\hline
\end{tabular}

Table 4. Thermophysical properties

\begin{tabular}{ccc} 
Properties & IL01 & IL02 \\
Total energy, (kcal/mol) & -59316.70985 & -52566.7697 \\
Entropy, (kcal/mol-deg) & 0 & 0 \\
Free energy, (kcal/mol) & -59316.70985 & -52566.7697 \\
Heat capacity, (kcal/mol-deg) & 0 & 0 \\
Dipole moment, (D) & 9.187 & 0 \\
RMS gradient, (kcal/mol) & 0.0992 & 0.05182 \\
Binding energy, (kcal/mol) & -2578.0250 & -2500.6007 \\
Heat of Formation & -83.9270 & -66.0617 \\
Electronic energy, (kcal/mol) & -343586.3093 & -282458.2046 \\
Nuclear energy, (kcal/mol) & 284269.5994 & 229891.4349 \\
\hline
\end{tabular}

Table 5. Data for entropy and heat capacity with variety temperature

\begin{tabular}{ccccccc} 
& \multicolumn{2}{c}{$273 \mathrm{~K}$} & \multicolumn{2}{c}{$298 \mathrm{~K}$} & \multicolumn{2}{c}{$323 \mathrm{~K}$} \\
& Entropy & $\begin{array}{c}\text { Heat capacity, } \\
\text { (kcal/mol-deg) }\end{array}$ & Entropy & $\begin{array}{c}\text { Heat capacity, } \\
\text { (kcal/mol-deg) }\end{array}$ & Entropy & $\begin{array}{c}\text { Heat capacity, } \\
\text { (kcal/mol-deg) }\end{array}$ \\
IL01 & 0.1211 & 0.0515 & 0.1260 & 0.0555 & 0.1308 & 0.0595 \\
IL02 & 0.1201 & 0.0433 & 0.1242 & 0.0466 & 0.1283 & 0.0499 \\
\hline
\end{tabular}

\section{Vibrational spectrum}

The existence of the functional group in the molecule can be identified through the vibrational spectroscopy. Since morpholinium cation creates ionic liquids in combination with various types of inorganic anion, and then there is a large spectrum between 3000 to $3500 \mathrm{~cm}^{-1}$ for NH levels of morpholinium cation which is taken from Figure 3 and Table 6 indicates the calculation condition. It is also found available for 2500 to $2200 \mathrm{~cm}^{-1}$ spectrums for $\mathrm{CH}$ of morpholinium cation. 


\section{UV-visible Spectrum}

The main aim of UV visible spectrum is to determine the ultra violet region and visible region. The ultraviolet region falls in the range between $190-380 \mathrm{~nm}$, the visible region fall between $380-750 \mathrm{~nm}$. The $\sigma$ to $\sigma^{*}$ transition requires an absorption of a photon with a wavelength which does not fall in the UV-vis range. From Figure 4, it is shown that the both of visible and ultravisible region are shown and Table 7 is the required condition for calculation.

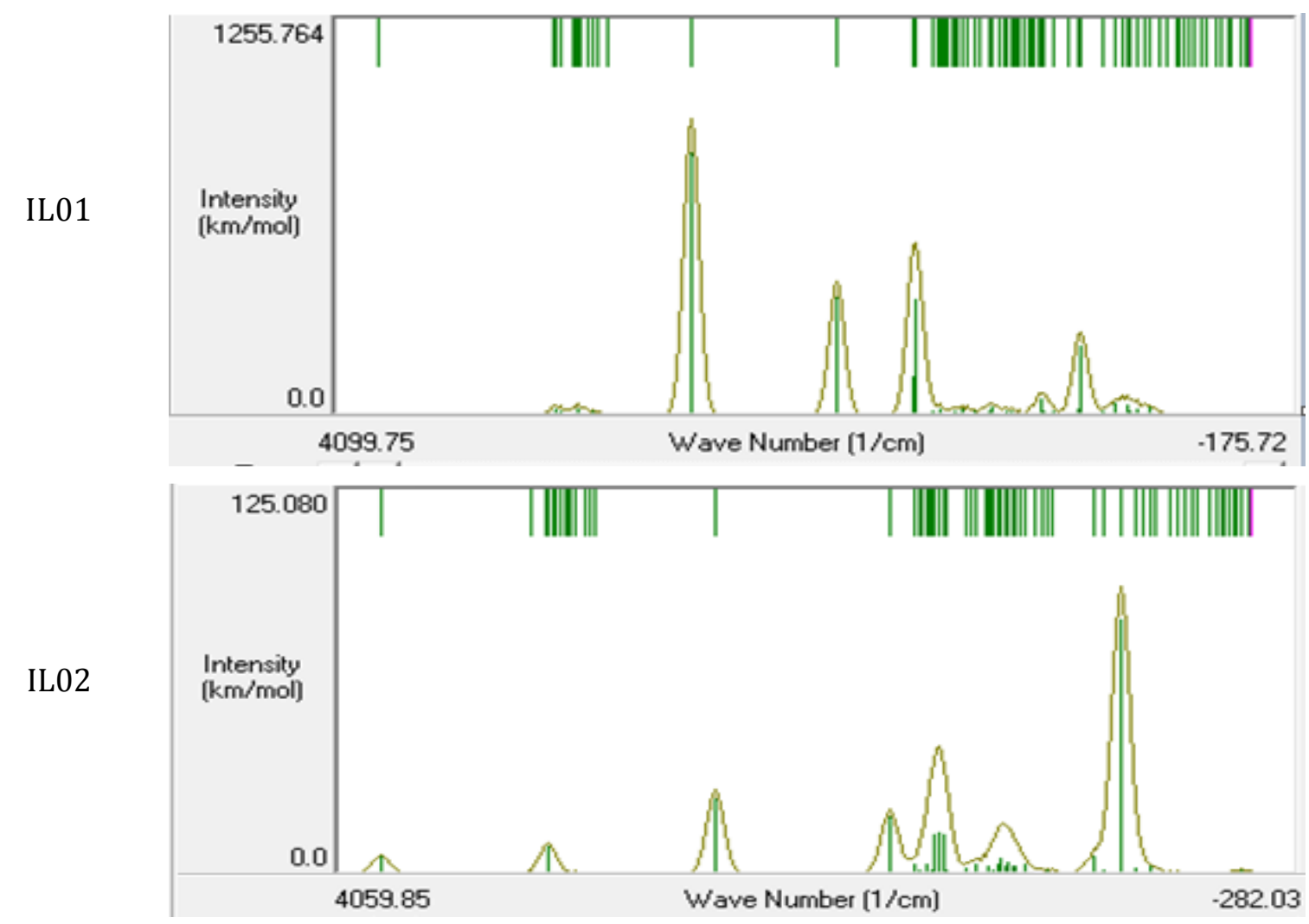

Figure 3. Vibrational spectrum

Table 6. Data of vibrational spectrum

$\begin{array}{cccccc} & \text { Normal Mode } & \text { Degeneracy } & \text { Frequency } & \text { Intensity } & \text { Symmetry } \\ \text { IL01 } & 1 & 1 & 18.62 & 0.752 & 1 \mathrm{~A} \\ \text { IL02 } & 1 & 1 & -84.67 & 0.506 & 1 \mathrm{~A}\end{array}$

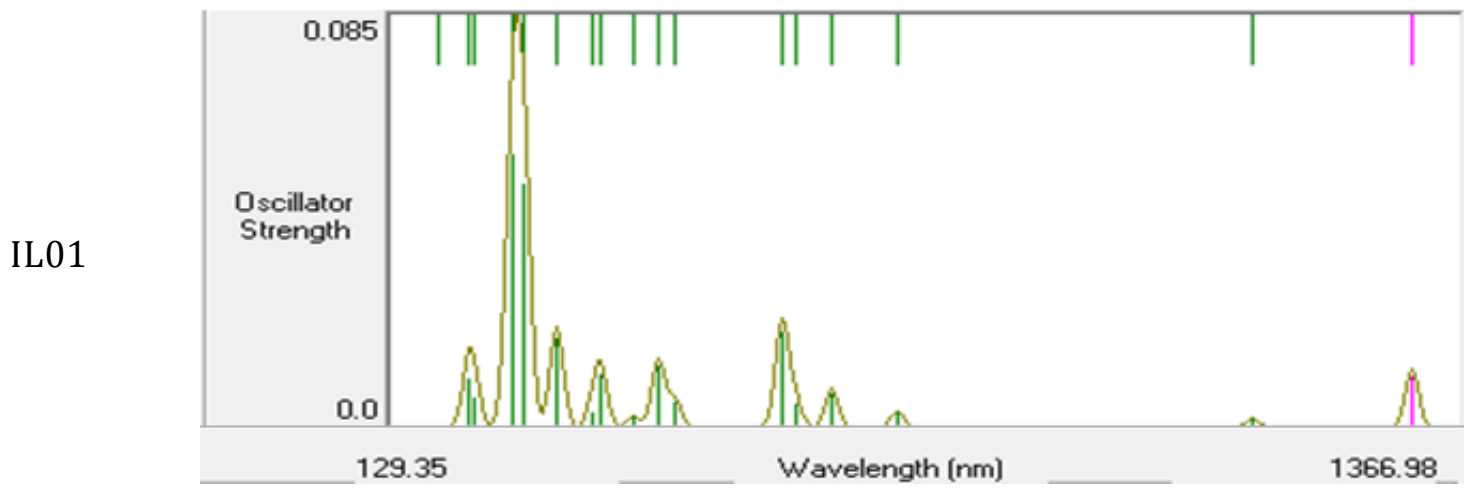


IL02

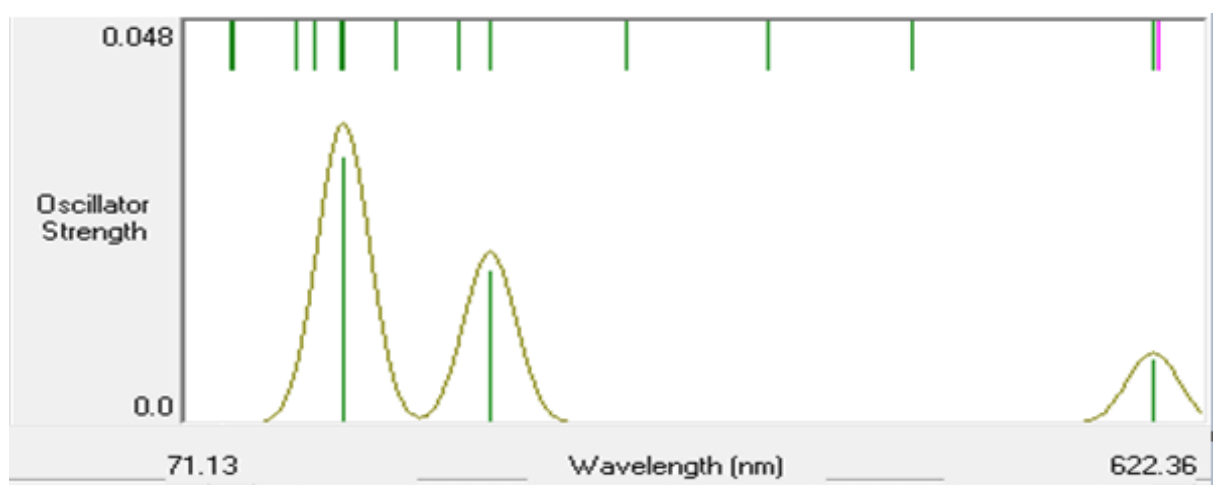

Figure 4. UV visible spectrum

Table 7. Data of UV visible spectrum

\begin{tabular}{cccccc} 
& Transition & Degeneracy & Spin Multiplicity & Wavelength & Oscillator Strength \\
IL01 & 1 & 1 & Doublet & 1310.72 & 0.0 \\
IL02 & 1 & 1 & Singlet & 597.30 & 0.0 \\
\hline
\end{tabular}

\section{Biological activity of optimized molecules}

The distribution electrostatic potential due to $3 D$ mapped structure

The biological activity of a compound can be estimated on the basis of the energy difference $\Delta \mathrm{E}$ frontier orbitals. This difference, $\Delta \mathrm{E}$ represents the electronic excitation energy which is possible in a molecule shown in Table 8 and Figure 5 . According the mechanism of antimicrobial activity and antimicrobial agents of bioactive molecules, the positive charge end of molecules is responsible to damage the plasma membrane of pathogens [23]. To kill to pathogens, the region of molecules was used the positive charge area of the molecule. In this case, the most important factors is explained that the higher surface area having positive charge is considered as the high antimicrobial activity.

Here, E1=Electrostatic potential energy in positive value, E2=Electrostatic potential energy in negative value and $\Delta \mathrm{E}=$ Electrostatic potential energy difference of two level.

Quantitative structure-activity relationships (QSAR)

A negative value of $\log P$ indicates the hydrophilicity and positive Log $\mathrm{P}$ indicates the hydrophobicity that plays an important role in biochemical interactions and bioactivity. Hydrophobic drugs tend to be more toxic because, in general, are kept longer, have a wider distribution in the body, are somewhat less selective in their binding to molecules and finally are often extensively metabolized. The molecule shows hydrophobicity which properties are given in Table 9.

\section{Calculation of PIC50}

The correlation between the biological activity and descriptor is developed by Almi et al., 2014 [24] for the PIC50 value calculation from the Hyperchem simulation value that is given in following equation and listed Table 10.

$$
\begin{gathered}
\mathrm{PIC50}=3.028-0.542 \log \mathrm{P}+0.352 \mathrm{HE}-1.272 \mathrm{Pol}+0.863 \mathrm{MR}-0.038 \mathrm{MV} \\
-0.024 \mathrm{MW}+19.120 \mathrm{q} 01+0.024 \mathrm{SAG}
\end{gathered}
$$




\section{IL01}

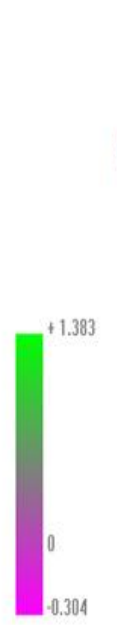

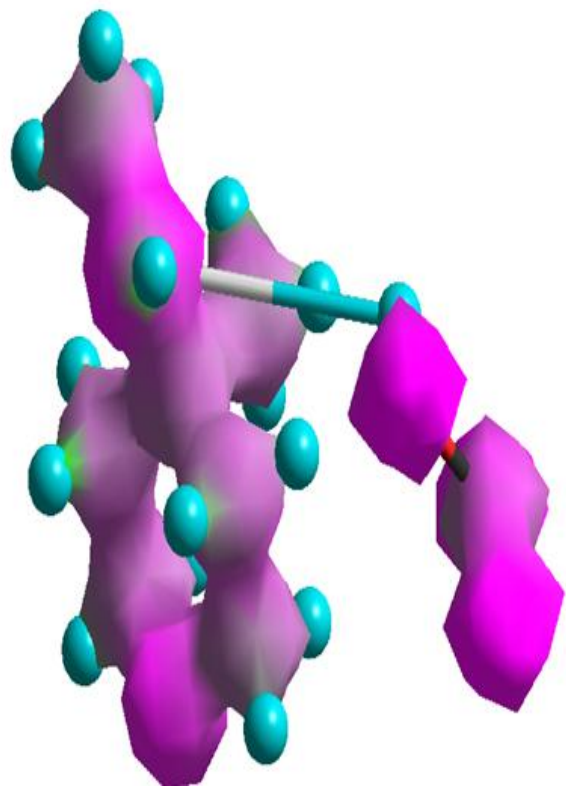

IL02

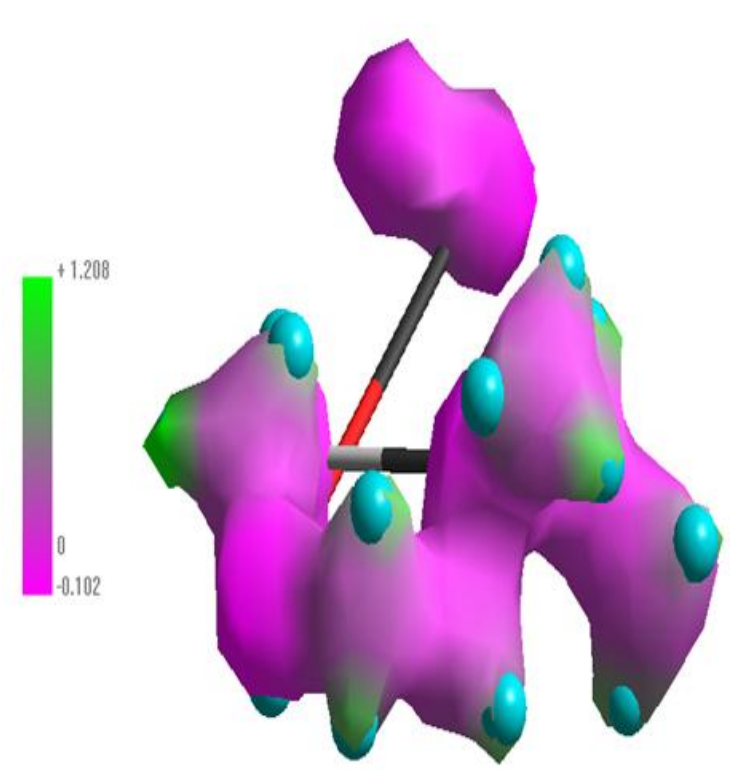

Figure 5. The 3D geometry of the distribution electrostatic potential

Table 8. Data of electrostatic potential energy difference of two levels

\begin{tabular}{ccc} 
& IL01 & IL02 \\
E1 & +1.383 & +1.208 \\
E2 & -0.304 & -0.102 \\
$\Delta \mathrm{E}=\mathrm{E} 2-\mathrm{E} 1$ & -1.687 & -1.310 \\
\hline
\end{tabular}

Table 9. Data for QSAR study

\begin{tabular}{ccc} 
& IL01 & IL02 \\
Partial charge (e) & 0.0 & 0.0 \\
Surface Area(grid), & 377.63 & 416.49 \\
Volume, $\AA^{3}$ & 596.90 & 645.71 \\
Hydration Energy kcal/mol & -7.75 & -14.53 \\
Log P & 0.51 & 1.48 \\
Refractivity $\AA^{3}$ & 43.94 & 44.71 \\
Polarizibility, $\AA^{3}$ & 17.05 & 16.42 \\
Mass (amu) & 199.22 & 176.22 \\
\hline
\end{tabular}

Table 10. Data of PIC50

\begin{tabular}{ccc} 
& IL01 & IL02 \\
PIC50 & -2.1441 & -3.9607 \\
\hline
\end{tabular}


Here, HE=Hydration Energy, Pol= Polazibility, MR= Molecular Refractivity, Log $\mathrm{P}=$ Partition coefficient, $\mathrm{MV}=$ Molar Volume, $\mathrm{MW}=$ Molar Weight, $\mathrm{SAG}=$ Surface Area Grid, $\mathrm{q} 01=$ atomic net charges .

\section{Conclusion}

To sum up, it is concluded that the biological chemistry, chemical reactivity and molecular orbital study like QSAR, HOMO, LUMO, HOMO-LUMO gap, ionization potential, electron affinity, and electrostatic potential in case of the charge distribution in molecule were optimized. From the data it is found that both of anion show highly bioactivity in case of PIC50 calculation and nitrate is more active than nitrite anion for morpholinium cation.

\section{Acknowledgment}

I am thankful to my professor Dr. Parimal Bala and Professor Dr. Dipika Rani sarker, Department of Physics, Jagannath University, Dhaka for their inspiration to carry on my research.

\section{ORCID}

M. J. Islam (D): 0000-0002-4125-8222

A. Kumer (D): 0000-0001-5136-6166

N. Sarker (D): 0000-0003-2760-0113

S. Paul (D: 0000-0001-7739-4018

\section{References}

[1]. L. Martini, J.L. Whistler, Curr. Opin Neurobiol., 2007, 17, 556-564.

[2]. W.D. Bowen, S. Gentleman, M. Herkenham, C.B. Pert, Proc. Natl. Acad. Sci. U. S. A., 1981, 78, 4818-4822.

[3]. T.J. Coderre, A.L. Vaccarino, R. Melzack, Brain Res., 1990. 535(1): p. 155-158. [4]. T.J. Coderre, J. Katz, A.L. Vaccarino, R. Melzack, Pain., 1993, 52259-285.
[5]. H.J. Groenewegen, F.T. Russchen, J. Comp. Neurol., 1984, 223, 347-367.

[6]. D. Van Der Kooy, R.F. Mucha, M. O'Shaughnessy, P. Bucenieks, Brain Res., 1982, 243, 107-117.

[7]. D.E. Monti, E. Egiziano, S. Burgalassi, P. Chetoni, C. Chiappe, A. Sanzone, S. Tampucci, Int. J. Pharm., 2017, 516, 45-51.

[8]. I. Newington, J.M. Perez-Arlandis, T. Welton, Org. Lett., 2007, 9, 5247-5250.

[9]. J.N.G. Pendleton, F. Brendan, Int. J. Antimicrobial Agents, 2015, 46, 131-139.

[10]. A.P.C. Abbott, G. Capper, D.L. Davies, R.K. Rasheed, Chem, A Eur. J., 2004, 10, 37693774.

[11]. W.L. Hough, S. Marcin, H. Rodríguez, R.P. Swatloski, S.K. Spear, D.T. Daly, J. Pernak, J.E. Grisel, R.D. Carliss, M.D. Soutullo, J.H. Davis, R.D. Rogers, New J. Chem., 2007, 31, 1429-1436.

[12]. M.I. Hossain, A. Kumer, S.H. Begum, Asian J. Phys. Chem. Sci., 2018, 3, 1-9.

[13]. M.I. Hossain, A. Kumer, Asian J. Phys. Chem. Sci., 2017, 3, 1-13.

[14]. M.I. Hossain, A. Kumer, Asian J. Phys. Chem. Sci., 2018, 3, 1-10.

[15]. R. Car, P. Mark, Phys. Rev. Lett., 1985, $55,2471$.

[16]. W. Yang, Phys. Rev. Lett., 1991, 66, 1438.

[17]. A.H. Shapiro, The Dynamics and Thermodynamics of Compressible Fluid Flow, In Two Volumes. 1953, Wiley.

[18]. M.P. Andersson, P. Uvdal, J. Phys. Chem. A, 2005, 109, 2937-2941.

[19]. C.J. Horowitz, D.B. Serot, Nuclear Phys. A, 1981, 368, 503-528.

[20]. A. Kumer, M. Sarker, S. Paul, Int. J. Chem. Technol., 2019, 3, 26-37

[21]. A. Kumer, M. Sarker, S. Paul, A. Zannat, Adv. J. Chem. A, 2019, 2, 190-202.

[22]. A. Howard, J. McIver, J. Collins, Hyperchem computational chemistry. Hypercube Inc., Waterloo, 1994.

[23]. L. Timofeeva, N. Kleshcheva, Appl. Microbial. Biotechnol., 2011, 89, 475-492. 
[24]. Z. Almi, S. Belaidi T. Lanez, T. Noureddine, Int. Lett. Chem., Phys. Astronomy, 2014, 37, 113-124.

How to cite this manuscript: Mohammad Jahidul Islam, Ajoy Kumer*, Nuruzzaman Sarker, Sunanda Paul, Afroza Zannat. The Prediction and Theoretical Study for Chemical Reactivity, Thermophysical and Biological Activity of Morpholinium Nitrate and Nitrite Ionic Liquid Crystals: A DFT Study. Adv. J. Chem. A, 2019, 2(4), 316-326. 